\title{
Optically Gated Terahertz-Field-Driven Switching of Antiferromagnetic CuMnAs
}

\author{
J.J.F. Heitz, ${ }^{1,2}$ L. Nádvorník $\odot,{ }^{1,2,3}$ V. Balos, ${ }^{1,2}$ Y. Behovits $\odot,{ }^{1,2}$ A.L. Chekhov ${ }^{1,2}$ T.S. Seifert, ${ }^{1,2}$ \\ K. Olejník, ${ }^{4}$ Z. Kašpar®, ${ }^{3,4}$ K. Geishendorf, ${ }^{3,4}$ V. Novák®, ${ }^{4}$ R.P. Campion, ${ }^{5}$ M. Wolf, ${ }^{2}$ \\ T. Jungwirth $\odot,{ }^{4,5}$ and T. Kampfrath $\oplus^{1,2}$ \\ ${ }^{1}$ Department of Physics, Freie Universität Berlin, 14195 Berlin, Germany \\ ${ }^{2}$ Department of Physical Chemistry, Fritz Haber Institute of the Max Planck Society, 14195 Berlin, Germany \\ ${ }^{3}$ Faculty of Mathematics and Physics, Charles University, 12116 Prague, Czech Republic \\ ${ }^{4}$ Institute of Physics, Academy of Sciences of the Czech Republic, v.v.i., 16200 Prague, Czech Republic \\ ${ }^{5}$ School of Physics and Astronomy, University of Nottingham, Nottingham NG7 2RD, UK
}

(Received 1 June 2021; revised 16 September 2021; accepted 24 November 2021; published 20 December 2021)

\begin{abstract}
We show scalable and complete suppression of the recently reported terahertz-pulse-induced switching between different resistance states of antiferromagnetic CuMnAs thin films by ultrafast gating. The gating functionality is achieved by an optically generated transiently conductive parallel channel in the semiconducting substrate underneath the metallic layer. The photocarrier lifetime determines the timescale of the suppression. As we do not observe a direct impact of the optical pulse on the state of CuMnAs, all observed effects are primarily mediated by the substrate. The sample region of suppressed resistance switching is given by the optical spot size, thereby making our scheme potentially applicable for transient low-power masking of structured areas with feature sizes of about $200 \mathrm{~nm}$ and even smaller.
\end{abstract}

DOI: 10.1103/PhysRevApplied.16.064047

\section{INTRODUCTION}

Information storage using antiferromagnetic (AFM) order has potential advantages compared to ferromagnets. (i) No stray field is present, allowing for a higher integration density of memory cells [1]. (ii) Potentially fast switching of the Néel vector is possible because angular momentum transfer to the crystal lattice, which is usually slow in insulators [2], is not required. In addition, magnon frequencies extend into the terahertz range even for small wave vectors $[3,4]$. Finally, (iii) robust retention of information is guaranteed because of the negligible impact of magnetic fields typically up to units or even tens of tesla [5-7].

The last feature also poses a challenge because strong quasistatic magnetic fields may be required to manipulate AFM order [7]. Recently, however, electromagnetic pulses from kilohertz up to optical frequencies [5] have been shown to be a powerful alternative tool. Here, coherent switching of the spatially uniform Néel vector is driven by the torque of an (effective) magnetic field, which may

\footnotetext{
*nadvornik@karlov.mff.cuni.cz
}

Published by the American Physical Society under the terms of the Creative Commons Attribution 4.0 International license. Further distribution of this work must maintain attribution to the author(s) and the published article's title, journal citation, and DOI. Open access publication funded by the Max Planck Society. derive directly from the magnetic field of a terahertz pulse [8], the electric field of a millisecond kilohertz [9-11] or a picosecond terahertz pulse [12], or from a rectified version of the electric field of a terahertz [13] or optical laser pulse [14]. For CuMnAs, both $90^{\circ}$ (field-alignment-dependent) [9] and $180^{\circ}$ (field-polarity-dependent) [11] reorientation switching of the Néel vector have been observed. In the latter experiments, the broken inversion symmetry of the AFM materials CuMnAs [9-11] and $\mathrm{Mn}_{2} \mathrm{Au}[15,16]$ is an important prerequisite [17].

In contrast, incoherent switching of AFM order typically arises from heating, which has been induced through electric currents $[6,18,19]$ or light $[6,20]$. For CuMnAs devices [such as in Fig. 1(a)], reproducible electrical and optical switching between different resistance states has been shown. The switched state persisted for tens of seconds at room temperature and much longer at lower temperatures $[6,21]$. Incoherent switching of CuMnAs is accompanied by domain fragmentation and features a multistate character $[6,18,19]$, potentially permitting even neuromorphic operations. Interestingly, switching of CuMnAs has also been achieved by free-space subpicosecond electric-field transients driving charge currents at terahertz frequencies [see Fig. 1(b)] [22]. This process is very likely dominated by incoherent domain fragmentation, too, because of the typical subsequent relaxation character.

Note that AFM CuMnAs thin films can be grown directly on common semiconductor substrates such as 
(a)

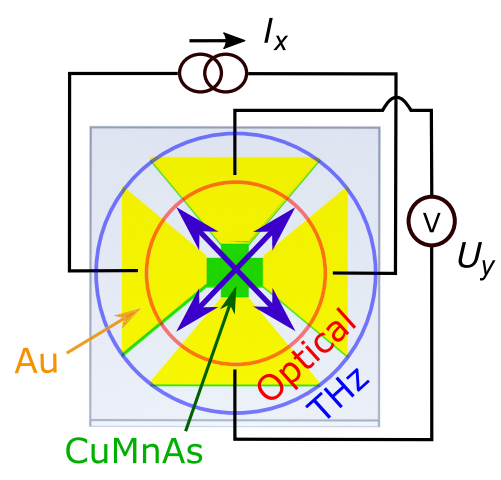

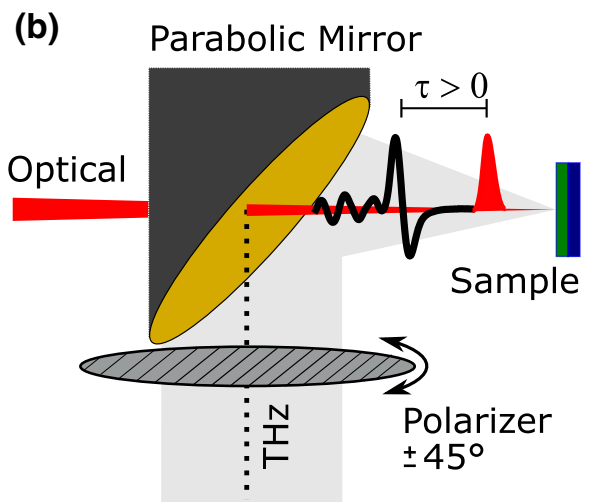

(c)

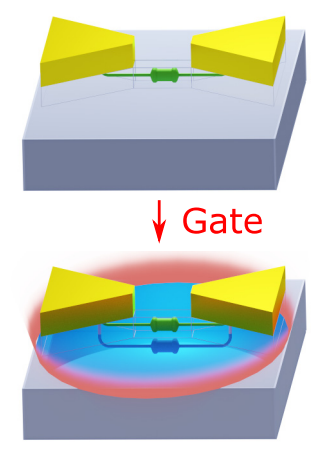

FIG. 1. Schematic of the experiment. (a) Sample structure (top view). A thin film of AFM CuMnAs (green crosslike area) is excited by a normally incident terahertz pulse with circular beam cross section (blue circle) and one of two perpendicular linear polarizations (indicated by blue arrows). The structure can be gated by an additional optical femtosecond laser pulse (red circle), which excites the CuMnAs layer and the underlying substrate. To measure the electrical anisotropy of the CuMnAs layer, a cross of Au contact pads (yellow layer) allows one to apply a read current $I_{x}$ and to detect the resulting transverse voltage $U_{y}$. The Au cross also enhances the incident terahertz field. (b) Schematic of the setup showing how terahertz radiation and optical gate pulses are focused on the sample surface. The two orientations of the polarizer define the two perpendicular linear terahertz polarization states as shown in panel (a). (c) Principle of gating by modulating the substrate conductance. For simplicity, only one arm of the cross is shown. Without any optical excitation, the two contact pads are electrically connected through the conductance of CuMnAs (green resistor in upper sketch). Upon excitation, the optical gate generates mobile charge carriers in the substrate which shunt the incident terahertz field (blue parallel resistor in lower sketch) and, thus, reduce the total terahertz field inside the CuMnAs layer.

$\mathrm{Si}$, GaAs, or GaP [6,23], which enable additional functionalities. An important example is the variation of the shunt resistance of the substrate, which may modulate the switching process in CuMnAs [see Fig. 1(c)]. A reduction of the substrate resistance can be achieved straightforwardly by excitation with an optical femtosecond gate pulse that generates quasifree electrons and holes by both direct and indirect optical transitions [24-26]. Such transient conductivities have already been successfully exploited to modulate the response of terahertz metamaterials [27] or even generate transient metamaterials by structured illumination $[28,29]$. In our setup, the reduced substrate resistance shorts the subsequently arriving terahertz electric field and, thus, should reduce the current driven in the AFM layer [see Fig. 1(c)], without the need for additional microstructuring. It remains to be shown whether this effect is sufficient to suppress the switching process and whether the direct impact of the optical pulse on the CuMnAs layer is negligible.

In this work, we study the interplay between terahertzdriven switching of a CuMnAs thin film and the ultrafast optical excitation of the semiconducting substrate. For optically absorbing substrates, we find that the optical pump with powers smaller than the optical writing threshold found in Ref. [6] can efficiently modulate and even completely suppress the switching process driven by the subsequently incident terahertz field but does not destroy information already written in the CuMnAs. Our observation is consistent with a model based on screening of the terahertz field by the optically induced free charge carriers. Our results demonstrate that integrating AFM devices with semiconductors enables functionalities with technological relevance.

\section{SETUP AND SAMPLES}

A schematic of our experiment is shown in Fig. 1(b). An AFM CuMnAs thin film [Fig. 1(a)] grown on top of a semiconducting substrate is excited by a pair of incident pulses at a repetition rate of $1 \mathrm{kHz}$ : first the optical gate pulse and, after a delay time $\tau$, the terahertz switching pulse with polarization directions at $+45^{\circ}$ or $-45^{\circ}$ with respect to the vertical axis. The two terahertz polarizations allow one to switch the AFM device between defined resistance states [22]. The impact of the excitation is probed by the continuous electrical measurement of the transverse sample resistance via Au contact pads [Fig. 1(a)].

\section{A. Samples}

Two kinds of samples are studied: thin films of AFM CuMnAs (thickness $50 \mathrm{~nm}$, sheet conductance $40 \mathrm{mS}$ on $\mathrm{GaAs}$ and $50 \mathrm{mS}$ on $\mathrm{GaP}$ ) on a substrate (thickness $500 \mu \mathrm{m}$ ) of GaAs (optical band gap $1.43 \mathrm{eV}$ at room temperature) and $\mathrm{GaP}(2.26 \mathrm{eV})$ [30,31]. These substrate materials allow us to control the amount of energy that is absorbed in the substrate from the optical gate pulse by varying the gate wavelength.

As indicated by Fig. 1(a), the CuMnAs layer is patterned and partly etched out to form a cross-shaped structure (side length of the square central region $2-4 \mu \mathrm{m}$ ). The Au contact pads (length $330 \mu \mathrm{m}$, width $210 \mu \mathrm{m}$ ), which consist 
of an Au layer (thickness $80 \mathrm{~nm}$ ) deposited on a Cr wetting layer $(5 \mathrm{~nm})$, are tapered toward the cross for the last third of the length and serve two purposes. First, they act as an antenna that enhances the incident terahertz electric field in the cross [blue arrows in Fig. 1(a)] by a factor of typically around 20 [22]. Details of the enhancement determination and the terahertz field distribution in the CuMnAs film can be found in Ref. [22]. Second, the Au pads serve as contacts to detect the electrical anisotropy of the sample [Fig. 1(a)], since we apply a dc read current $I_{x}(1-2 \mathrm{~mA})$ along the horizontal bar of the cross pattern and measure the transverse voltage $U_{y}$ at a read-out rate of $5 \mathrm{~Hz}$. Variations $\Delta R_{x y}$ of the transverse resistance $R_{x y}=U_{y} / I_{x}$ quantify changes in the electrical anisotropy of the sample [22] induced by the terahertz switching and optical gate pulse.

\section{B. Terahertz setup}

Intense terahertz pulses [Fig. 1(b)] are generated by optical rectification of ultrashort optical laser pulses $(800 \mathrm{~nm}$, duration stretched to $80 \mathrm{fs}$, energy $6 \mathrm{~mJ}$, repetition rate $1 \mathrm{kHz}$ ) in a $\mathrm{LiNbO}_{3}$ crystal, providing terahertz pulses with peak electric fields up to $1 \mathrm{MV} / \mathrm{cm}$ and durations of approximately $1 \mathrm{ps}$ [32]. The polarization state of the terahertz beam is controlled by a wire-grid polarizer $\left( \pm 45^{\circ}\right)$ and combined with optical gate pulses from the same laser by a parabolic mirror with a hole in its center. Both beams are focused onto the sample, forming spots with diameters of $2 b_{\mathrm{THz}}=1100 \mu \mathrm{m}$ (terahertz) and $2 b_{\mathrm{opt}}=85 \mu \mathrm{m}$ at photon energy $\epsilon_{\mathrm{opt}}=1.55 \mathrm{eV}$, as characterized by the pinhole method [33]. The gate-beam width $2 b_{\text {opt }}=60 \mu \mathrm{m}$ at photon energy $\epsilon_{\mathrm{opt}}=3.1 \mathrm{eV}$ is estimated from the $1.55 \mathrm{eV}$ value using Gaussian beam optics. The parameters $b_{\mathrm{THz}}$ and $b_{\text {opt }}$ denote the radial distance from the beam axis at which the peak intensity of the Gaussian beam has reduced by a factor of $1 / e$. The fluences $F_{\mathrm{THz}}$ and $F_{\mathrm{opt}}$ are calculated as the pulse energy divided by $\pi b_{\mathrm{THz}}^{2}$ and $\pi b_{\mathrm{opt}}^{2}$, respectively. With this definition, $F_{\mathrm{THz}}$ and $F_{\mathrm{opt}}$ are identical to the local peak fluence in the center of the Gaussian beam.

The fluences are controlled by a combination of optical wave plates with polarizers and neutral-density filters and the optical pump power incident on the $\mathrm{LiNbO}_{3}$ crystal. An example of a typical terahertz waveform (spectrum 0.3-2.5 THz, peak at $1 \mathrm{THz}$ ) and a schematic of an optical pulse are shown in Fig. 1(b). While the duration of the terahertz electromagnetic transient is smaller than $1 \mathrm{ps}$, the duration of the optical gate pulse is approximately $40 \mathrm{fs}$.

The sample structure is excited by a train of pulse pairs at a repetition rate of $1 \mathrm{kHz}$. As indicated by Fig. 1(b), each pair consists of a terahertz switching pulse (polarization directions at $\pm 45^{\circ}$ ) and an optical gate pulse. The delay $\tau$ between optical and terahertz pulse can be set between -1 and $+1 \mathrm{~ns}$ by a mechanical translation stage. Here, a positive or negative $\tau$ means that the terahertz
TABLE I. Parameters for the parallel-photoconductor model for the two substrates used.

\begin{tabular}{lccc}
\hline \hline Parameter & GaAs & GaP & Source \\
\hline$n_{S}(1 \mathrm{THz})$ & 3.6 & 3.3 & Refs. [34-36] \\
$\begin{array}{l}\text { Band gap } \\
\quad(\mathrm{eV})\end{array}$ & 1.43 & 2.26 & Refs. [30,31] \\
Gate photon & 1.55 & 3.1 & \\
$\quad$ energy & & & \\
$\epsilon_{\mathrm{opt}}(\mathrm{eV})$ & & & \\
$n_{S}\left(\epsilon_{\mathrm{opt}}\right)$ & $3.68+0.09 i$ & $4.20+0.27 i$ & Ref. [37] \\
$n_{\mathrm{AFM}}\left(\epsilon_{\mathrm{opt}}\right)$ & $3.7+2.0 i$ & $2.5+2.2 i$ & Ref. [38] \\
$T_{\mathrm{opt}}(\%)$ & 14 & 2.7 & Appendix A \\
$G_{\mathrm{AFM}}(\mathrm{mS})$ & 40 & 50 & Ref. [22], Fig. 5 \\
$2 b_{\mathrm{opt}}(\mu \mathrm{m})$ & 85 & 60 & Sec. II \\
$F_{\mathrm{opt}}^{\mathrm{max}}$ & 5.6 & 1.8 & Sec. II \\
$\left(\mathrm{mJ} / \mathrm{cm}^{2}\right)$ & & & \\
\hline \hline
\end{tabular}

${ }^{\text {a }}$ The transmittance $T_{\text {opt }}$ of the optical gate beam through the CuMnAs is calculated as described in the main text.

pulse arrives after or before the optical pulse at the sample, respectively [see Fig. 2(d)]. We emphasize that the optical fluences used here are at least 1 order of magnitude weaker than the fluence that would be needed to switch the AFM directly [6]. The experiments are conducted at room temperature under ambient conditions. More details of the optical and electrical setup can be found in the Supplemental Material [33].

\section{RESULTS}

\section{A. Typical data}

Figure 2 depicts a typical example of the measured transverse resistance $R_{x y}$ of CuMnAs on a GaAs substrate versus real time. The width of the shown time windows ranges from $40 \mathrm{~s}$ [Fig. 2(a)] to over $300 \mathrm{~s}$ [Fig. 2(b)] to $2000 \mathrm{~s}$ [Fig. 2(c)]. The fluence of the terahertz and optical pulse trains is set to $F_{\mathrm{THz}}=0.73 F_{\mathrm{THz}}^{\max }$ and $F_{\mathrm{opt}}=$ $0.006 F_{\mathrm{opt}}^{\max }$, respectively. The maximum terahertz fluence is $F_{\mathrm{THz}}^{\max }=62 \mu \mathrm{J} / \mathrm{cm}^{2}$, and the maximum optical fluences at 1.55 and $3.1 \mathrm{eV}$ are given in Table I.

Figure 2(a) displays one switching cycle where the gate pulse arrives after the terahertz switching pulse ( $\tau=$ $-240 \mathrm{ps}$ ). The course of the transverse resistance signal $R_{x y}$ has the characteristic shape of a switching cycle as observed in previous works $[6,18,22]$. Exposure with $-45^{\circ}$-polarized terahertz pulses causes switching, while the absence of the terahertz pulses ("THz off") results in a relaxation of the signal toward a baseline. Exposure with $+45^{\circ}$-polarized terahertz pulses switches the sample resistance state into the opposite direction away from the baseline, to which the signal relaxes back again after removing the terahertz excitation.

Figure 2(b) shows an extended time window that contains two signal groups. Each of these groups contains four 


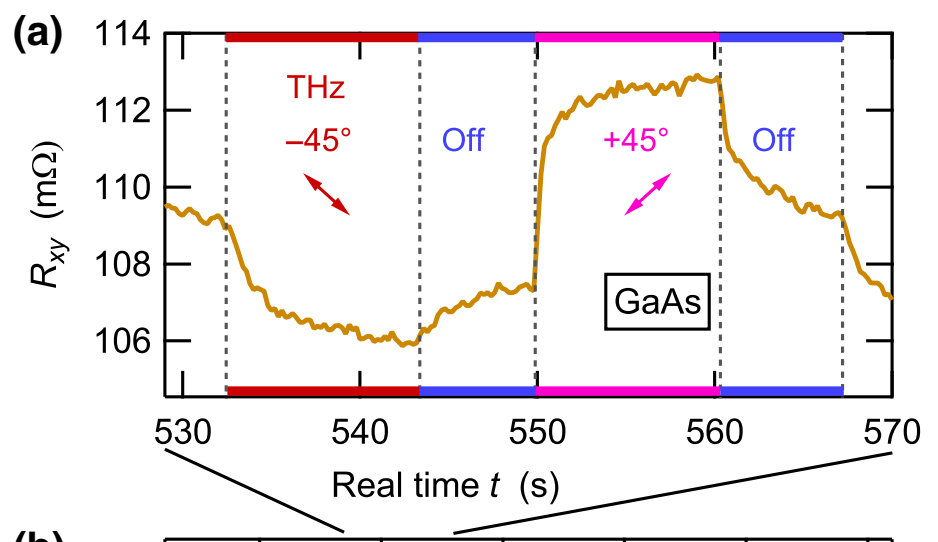

FIG. 2. Optically gated terahertz switching between resistivity states of a 3- $\mu \mathrm{m}$-large CuMnAs device on a GaAs substrate. Panels (a)-(c) show the dynamics of the transverse resistance $R_{x y}$ versus time $t$ in increasing degree of detail. (a) Typical dynamics of the transverse resistance induced by a train of terahertz pulses (fluence $F_{\mathrm{THz}}=$ $0.18 F_{\mathrm{THz}}^{\max }$, repetition rate $1 \mathrm{kHz}$ ) taken over one switching cycle $\left(-45^{\circ}\right.$ terahertz polarization, terahertz off, $+45^{\circ}$ terahertz polarization, terahertz off). (b) Temporal evolution of the transverse resistance when the terahertz pulses are preceded by synchronized optical gate pulses (photon energy $1.55 \mathrm{eV}, F_{\mathrm{opt}}=0.006 F_{\mathrm{opt}}^{\max }$, repetition rate $1 \mathrm{kHz}$ ). The switching-induced resistance change $\Delta R$ is indicated by the double arrow. Data points highlighted in black are averaged over, and the average of each group is indicated as a black dashed line. At real time $t<612 \mathrm{~s}$ (brown data), the optical excitation occurs after the terahertz switching pulse (terahertz-optical gate pulse delay $\tau<0 \mathrm{ps}$ ), whereas at $t>612 \mathrm{~s}$ (green data), optical excitation temporally overlaps with the terahertz pulse $(\tau \approx 0 \mathrm{ps})$. (c) The same data as in panel (b), but over a larger time window. The delay $\tau$, corresponding to each color-labeled block of data, is stated above the traces. (d) Resistance modulation $\Delta R$ as inferred from panel (c) versus delay $\tau$. Each data point is the mean of $\Delta R$ of the last three switching cycles per set of four (color coded) in (b). The error bars are the standard deviation of all data at $\tau<0$ to more adequately account for the long-term signal drifts. The red curve is a monoexponential fit with a relaxation time of $2.3 \mathrm{~ns}$. The maximum incident fluences are given by $F_{\mathrm{THz}}^{\max }=62 \mu \mathrm{J} / \mathrm{cm}^{2}$ and $F_{\mathrm{opt}}^{\max }=5.6 \mathrm{~mJ} / \mathrm{cm}^{2}$ at $1.55 \mathrm{eV}$.
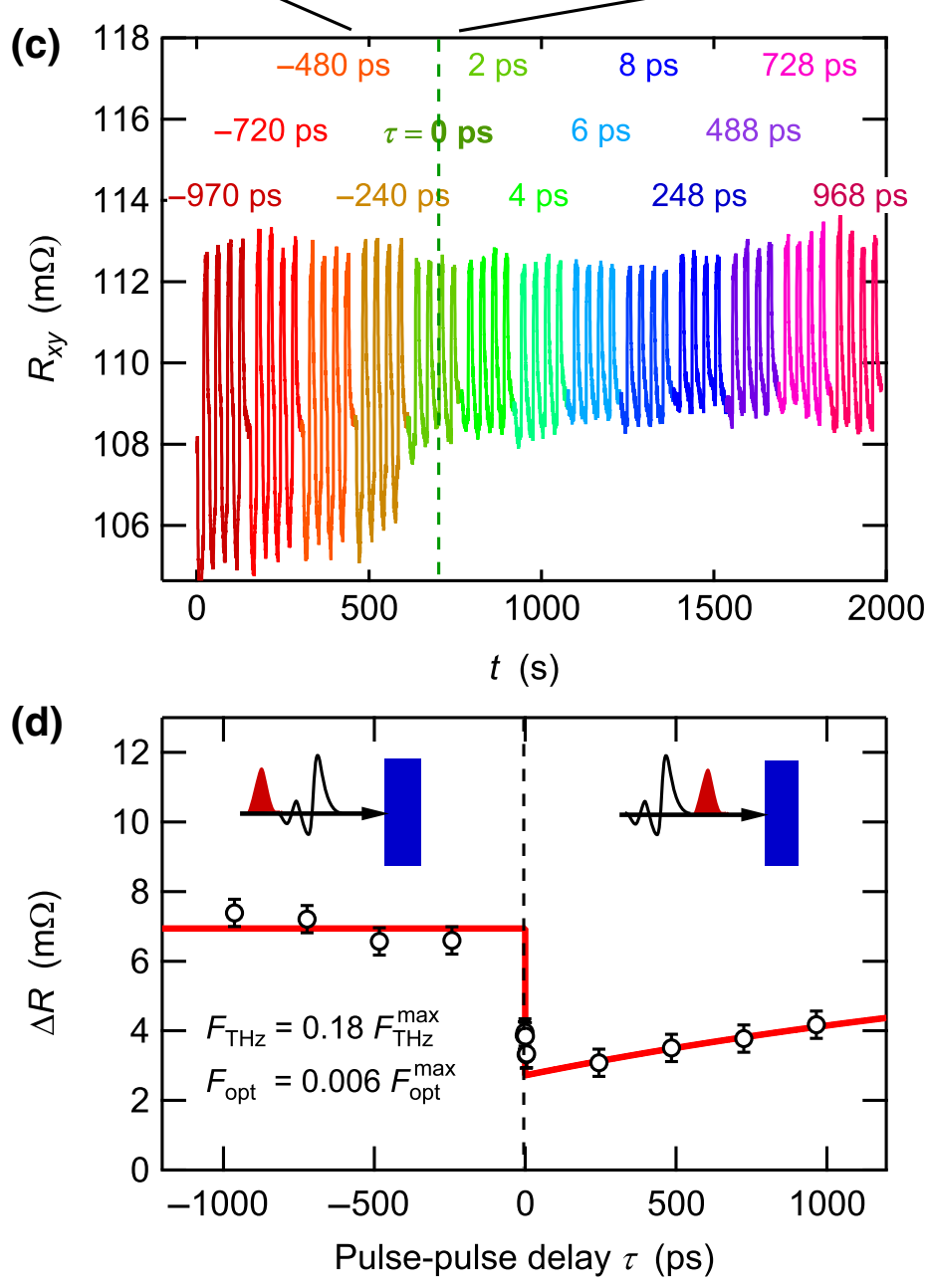
switching cycles and is marked by a different color. The first group of four (brown) is taken for $\tau=-240 \mathrm{ps}$ (gate arrives after terahertz pulse), whereas the second group (green) is measured for coinciding arrival times of terahertz and gate pulses $(\tau=0 \mathrm{ps})$. We observe a distinct reduction of the resistance modulation by a factor of about 2. The resistance modulation $\Delta R$ is the maximum variation of the transverse resistance $R_{x y}$ during the corresponding full switching cycle [see arrow in Fig. 2(b)].

Figure 2(c) displays an extended window of measurement time and, accordingly, a wider range of terahertz-gate delays $\tau$ than in Fig. 2(b). For negative delays $\tau<0$ (gate after terahertz pulse), there are no significant variations of the resistance modulation. At $\tau=0 \mathrm{ps}$, a reduction of resistance modulation is observed, which slightly recovers over the subsequent 1000 ps of optical-terahertz delay. To analyze this behavior, we extract the resistance modulation from the last three cycles of each group of Fig. 2(c). Note that the first cycle is omitted from this analysis as it is affected by the baseline shift from the previous parameter set. The average of these three contrast values for each group yields one data point. As an estimate of the error bar, we take the standard deviation of all data for $\tau<0$.

The resulting $\Delta R$ versus pump-probe delay is shown in Fig. 2(d). The gate-induced reduction of the resistance modulation is significantly bigger than the error bar, which is estimated as the standard deviation of all data at $\tau<0$ and, thus, also accounts for the long-term signal drifts.

The gate induces a steplike decrease of the resistance modulation when the optical pulse starts preceding the terahertz pulse, followed by relaxation on a nanosecond timescale. The underlying dynamics can be well described by a Heaviside step function times a monoexponential decay with a time constant of 2.3 ns plus a constant background [see red curve in Fig. 2(d)].

\section{B. Impact of the gate pulse}

Figures 2(c) and 2(d) demonstrate that the optical gate is capable of suppressing the contrast of switching over one terahertz-driven switching cycle. Note that the optical gate (photon energy $1.55 \mathrm{eV}$ ) used in the measurements, shown in Fig. 2, can excite both the GaAs substrate (electronic band gap $1.43 \mathrm{eV}$ ) and the CuMnAs film.

To address the role of the substrate, we change the substrate to $\mathrm{GaP}$ (band gap $2.25 \mathrm{eV}$ ) and repeat the experiment with a gate photon energy below $[1.55 \mathrm{eV}$, Fig. 3(a)] and above [3.1 eV, Fig. 3(b)] the GaP band gap. For below-gap gate excitation [Fig. 3(a)], no significant change in the resistance modulation is observed relative to the error bars of the measurement [Fig. 2(a)]. Thus, the direct excitation of CuMnAs has a minor impact on the switching dynamics, which most likely also holds true for the samples grown on GaAs.
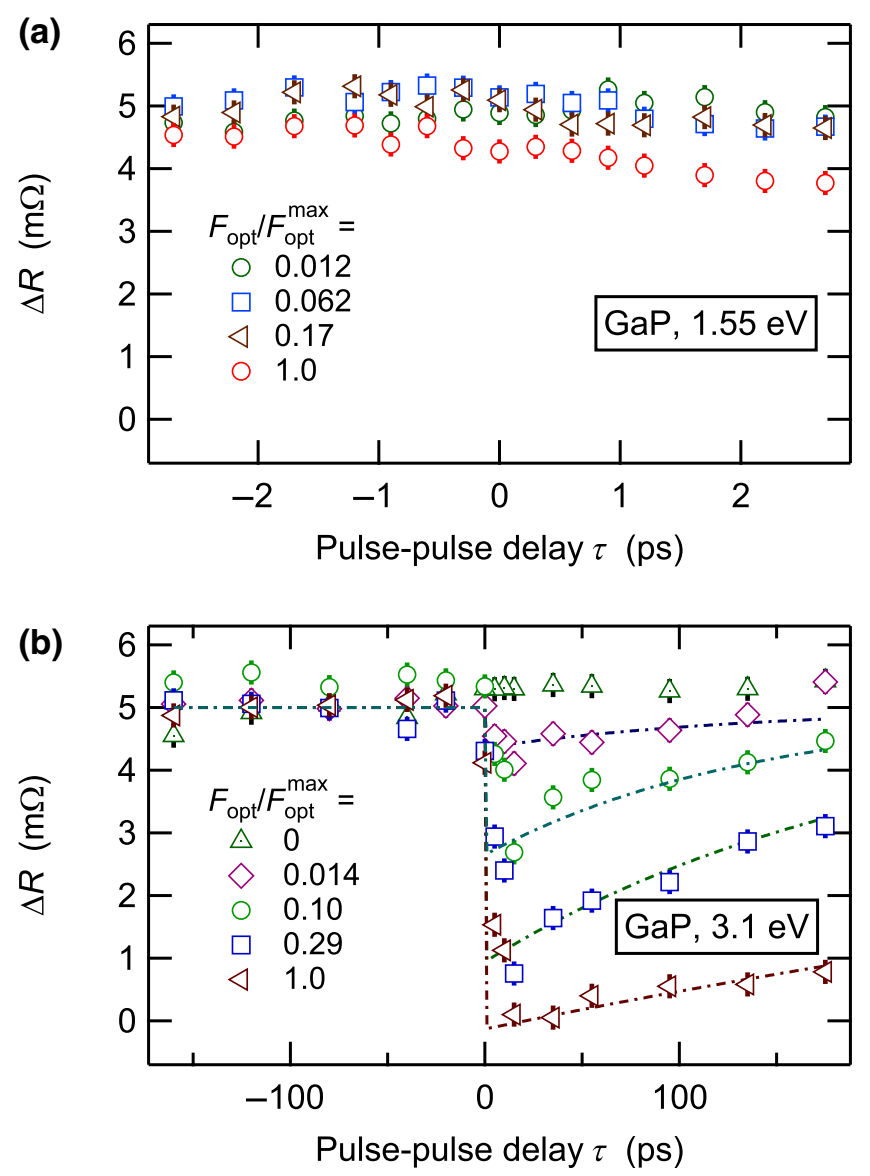

FIG. 3. Impact of the optical-gate photon energy and substrate on the terahertz switching of a $2-\mu \mathrm{m}$-large CuMnAs device on a GaP substrate. (a) Resistance modulation $\Delta R$ versus delay $\tau$ between optical gate and terahertz switching pulse for different gate-pulse fluences $F_{\text {opt }}$ with a gate photon energy of $1.55 \mathrm{eV}$, below the $2.25 \mathrm{eV}$ electronic band gap of GaP, and $F_{\mathrm{THz}}=1.0 F_{\mathrm{THz}}^{\mathrm{max}}$. (b) Same as panel (a), but for a gate photon energy of $3.1 \mathrm{eV}$, which is above the band gap of GaP. The data presented are the mean of each $\Delta R$ of the last three switching events per group as shown in Fig. 2(b). The error bars are the standard deviation of data at $\tau<0$. The maximum incident terahertz fluence is $F_{\mathrm{THz}}^{\max }=62 \mu \mathrm{J} / \mathrm{cm}^{2}$, while the maximum optical fluences $F_{\text {opt }}^{\max }$ amount to $5.6 \mathrm{~mJ} / \mathrm{cm}^{2}$ at $1.55 \mathrm{eV}$ and $1.8 \mathrm{~mJ} / \mathrm{cm}^{2}$ at $3.1 \mathrm{eV}$.

However, for above-gap gate excitation [Fig. 3(b)], a distinctly different behavior of the resistance modulation versus terahertz-optical delay is found. We observe a strong reduction of the resistance modulation that increases with gate fluence. At the maximum gate fluence of $F_{\mathrm{opt}}^{\mathrm{max}}$ at $3.1 \mathrm{eV}$, nearly complete suppression is achieved for delays below approximately 20 ps. We conclude that suppression of the switching signal is related to optical excitation of electronic transitions in the substrate underneath the CuMnAs device, while direct excitation of the CuMnAs and $\mathrm{Au}$ films (both at 1.55 and $3.1 \mathrm{eV}$ ) plays a minor 
role. The timescales on which the resistance modulation recovers are discussed further in the following.

\section{Power dependence}

To characterize how strongly the optically excited substrate modifies the terahertz excitation process, we measure the resistance modulation $\Delta R$ as a function of the terahertz fluence $F_{\mathrm{THz}}$ and the gate fluence $F_{\text {opt }}$ [Fig. 4(a)]. Without the optical gate pulses, we observe that the resistance modulation $\Delta R$ increases monotonically and strongly superlinearly with increasing terahertz fluence [red curve in Fig. 4(a)], consistent with the observations in Ref. [22].

In a second measurement, the terahertz fluence $F_{\mathrm{THz}}$ is held at the maximum available value $F_{\mathrm{THz}}^{\mathrm{max}}$, and the gatepulse train is switched on with a delay of $\tau=15 \mathrm{ps}$. We see that the resistance modulation $\Delta R$ decreases monotonically when the gate fluence $F_{\text {opt }}$ is increased [black curve in Fig. 4(a)]. At a gate fluence above $0.5 \%$ of $F_{\mathrm{opt}}^{\max }$ at $1.55 \mathrm{eV}$, $\Delta R$ is suppressed by more than $90 \%$. An analogous behavior of $\Delta R$ vs $F_{\mathrm{THz}}$ and $F_{\text {opt }}$ is found for the CuMnAs device on the GaP substrate for $3.1 \mathrm{eV}$ gate excitation [Fig. 4(c)], yet for significantly larger gate fluences, as discussed in the following section. Note that these observations demonstrate a high level of control over the resistance modulation by the gate pulse.

\section{D. "Parallel photoconductor" model}

A mechanism through which the optically excited substrate can modify the terahertz excitation process is the "parallel photoconductor" model, which is schematically shown in Fig. 1(c). In a qualitative picture, without the (a)

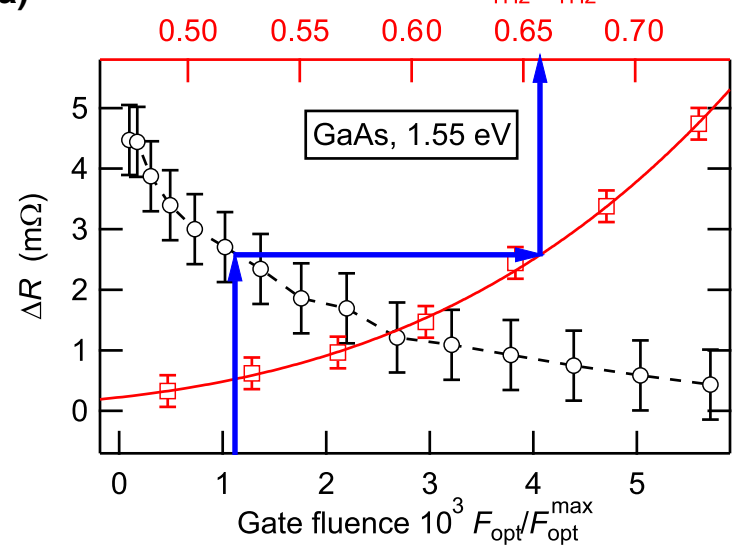

(b)

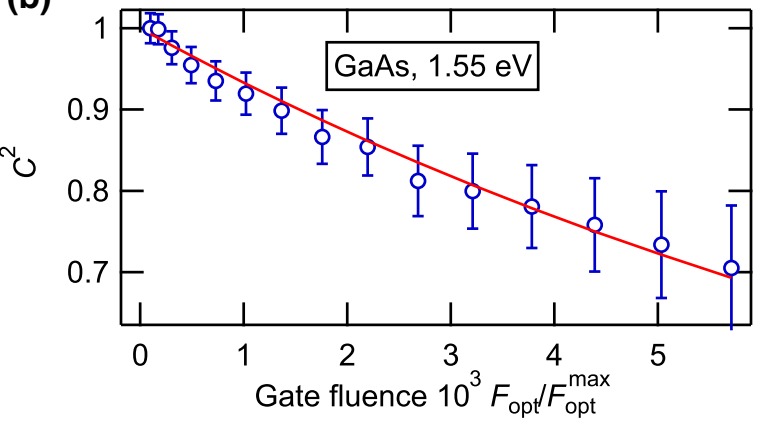

(c)

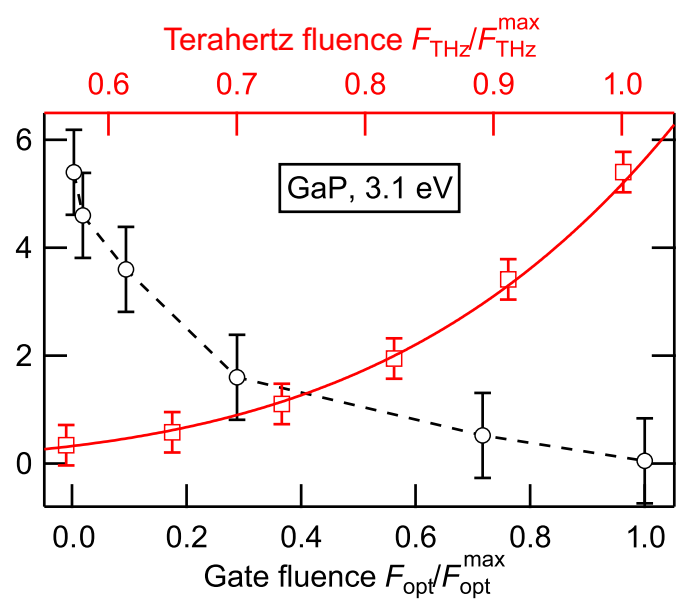

(d)

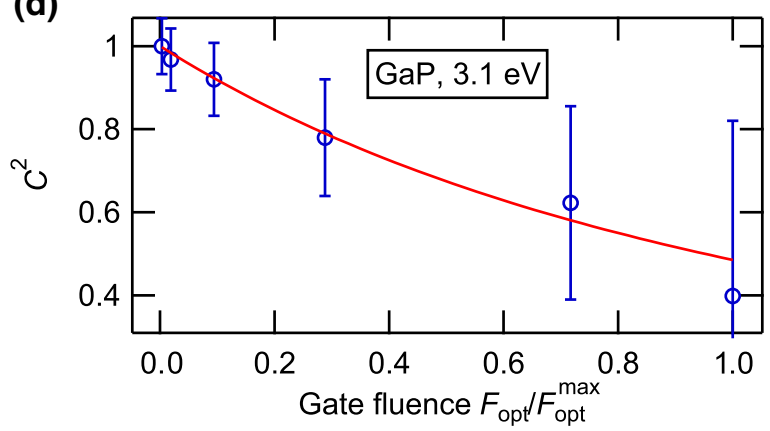

FIG. 4. Fluence dependence and optical gating mechanism. (a) Dependence of terahertz resistance modulation $\Delta R$ [see Fig. 2(b)] on the terahertz fluence $F_{\mathrm{THz}}$ (red symbols, top axis) for $F_{\mathrm{opt}}=0$ and on the gate fluence $F_{\mathrm{opt}}$ for $F_{\mathrm{THz}}=0.73 F_{\mathrm{THz}}^{\mathrm{max}}$ and a preceding optical gate pulse at $\tau=15$ ps (black symbols, bottom abscissa). The sample is a 4- $\mu \mathrm{m}$-large CuMnAs device on GaAs substrate, and the red solid line is a fit based on $F_{\mathrm{THz}}=a_{1}(\Delta R)^{a_{2}}+a_{3}$ with fit parameters $a_{1}, a_{2}, a_{3}$. (b) Experimentally determined (blue symbols) and modeled (red solid line) factor $C^{2}$ that quantifies how strongly the gate pulse attenuates the terahertz power arriving in the CuMnAs layer as a function of the gate fluence $F_{\text {opt }}$. The experimental values and their uncertainties are extracted from panel (a) using $C^{2}=F_{\mathrm{THz}}^{\mathrm{eq}} / F_{\mathrm{THz}}^{\mathrm{max}}$ (see blue arrows and text), whereas calculated values are derived from a simple parallel photoconductance model [see Fig. 1(c) and text]. (c) and (d) Same as panels (a) and (b), but for a 2- $\mu$ m-large CuMnAs device on a GaP substrate, a gate photon energy of $3.1 \mathrm{eV}, F_{\mathrm{THz}}=1.0 F_{\mathrm{THz}}^{\mathrm{max}}$, and a delay of $\tau=35 \mathrm{ps}$. The data presented are the mean of the three switching events per group as shown in Fig. 2(b). The error bars are the standard deviation of data at $\tau<0$. The maximum incident terahertz fluence is $F_{\mathrm{THz}}^{\max }=62 \mu \mathrm{J} / \mathrm{cm}^{2}$, while the maximum optical fluences $F_{\mathrm{opt}}^{\max }$ amount to $5.6 \mathrm{~mJ} / \mathrm{cm}^{2}$ at $1.55 \mathrm{eV}$ and $1.8 \mathrm{~mJ} / \mathrm{cm}^{2}$ at $3.1 \mathrm{eV}$. Note the different scaling of $F_{\text {opt }}$ in panels (a) and (b) versus (c) and (d). 
optical gate, the incident terahertz pulse drives a current exclusively through the AFM CuMnAs layer of conductance $G_{\mathrm{AFM}}$ [see Fig. 1(c) top]. However, if the terahertz pulse is preceded by an optical gate, the gate generates quasifree electrons and holes in the substrate, resulting in a transient electrically conducting layer with sheet conductance $\Delta G_{S}\left(F_{\mathrm{opt}}, \tau\right)$ [see Fig. 1(c) bottom]. The transient layer shunts the CuMnAs and, thus, reduces the current through the CuMnAs layer by a factor $C$.

We extract the dependence of $C$ on the gate fluence $F_{\text {opt }}$ from the data of Fig. 4(a). For a given $F_{\text {opt }}$ and at maximum terahertz fluence $\left(F_{\mathrm{THz}}=F_{\mathrm{THz}}^{\max }\right)$, we determine the equivalent terahertz fluence $F_{\mathrm{THz}}^{\mathrm{eq}}$ that is required to induce the same resistance modulation $\Delta R$ in the absence of the gate beam $\left(F_{\mathrm{opt}}=0\right)$. This procedure is illustrated by the three blue arrows in Fig. 4(a). It requires a continuous curve $\Delta R$ vs $F_{\mathrm{THz}}$, which is obtained from a fit based on $F_{\mathrm{THz}}=a_{1}(\Delta R)^{a_{2}}+a_{3}$ with fit parameters $a_{1}, a_{2}, a_{3}$. In this procedure, we also propagate the uncertainties (error bars) of the measured contrast $\Delta R$ vs $F_{\text {opt }}$, whereas the much smaller uncertainties of $\Delta R$ vs $F_{\mathrm{THz}}$ are neglected. As the terahertz power is proportional to the integrated squared terahertz field, the field-suppression factor $C$ is finally determined by the relationship $C^{2}=F_{\mathrm{THz}}^{\mathrm{eq}} / F_{\mathrm{THz}}^{\max }$. The resulting $C^{2}$ is plotted against $F_{\text {opt }}$ in Fig. 4(b). Starting from unity, $C^{2}$ decreases monotonically with increasing $F_{\text {opt }}$ from 0 to about $0.6 \%$ of $F_{\mathrm{opt}}^{\max }$ at $1.55 \mathrm{eV}$.

To test our parallel photoconductor model [Fig. 1(c)], we calculate $C$ using a simple model, in which we consider the device as an infinitely extended CuMnAs thin film between two half-spaces of air $(A)$ and the substrate $(S)$. As the penetration depth of the gate pulse is $750 \mathrm{~nm}$ for $\mathrm{GaAs}$ at $1.55 \mathrm{eV}$ and $116 \mathrm{~nm}$ for $\mathrm{GaP}$ at $3.1 \mathrm{eV}$, the thickness of the transiently conducting $S$ layer and the CuMnAs film $(50 \mathrm{~nm})$ is smaller than the skin depth $(>1 \mu \mathrm{m})$ of the terahertz field in the CuMnAs and substrate (see Appendix A). Therefore, the terahertz electric field is approximately constant across the thickness of the CuMnAs and the photoexcited substrate layer. This condition allows us to apply the Tinkham formula [39,40], which relates the incident terahertz electric field $E_{\text {inc }}$ to the electric field $E_{\mathrm{AFM}}$ inside the CuMnAs layer with the transmission coefficient

$$
t\left(\Delta G_{S}\right)=\frac{E_{\mathrm{AFM}}\left(\Delta G_{S}\right)}{E_{\mathrm{inc}}}=\frac{2 n_{A}}{n_{A}+n_{\mathrm{S}}+Z_{0} G_{\mathrm{AFM}}+Z_{0} \Delta G_{S}} .
$$

Here, $n_{A}$ and $n_{S}$ are the refractive indices of air and unexcited substrate, respectively, and $Z_{0} \approx 377 \Omega$ is the free-space impedance. Equation (1) shows that we can control the amplitude of the terahertz field $E_{\mathrm{AFM}}=t E_{\mathrm{inc}}$ inside CuMnAs by tuning the amplitude of $E_{\text {inc }}$ or by modifying the transient conductance $\Delta G_{\mathrm{S}}\left(P_{\mathrm{opt}}, \tau\right)$ of the photoexcited substrate layer through variation of the energy and time delay of the optical gate pulse. It follows that $\Delta G_{S}$ suppresses the field inside the CuMnAs by a factor of

$$
C=\frac{E_{\mathrm{AFM}}\left(\Delta G_{S}\right)}{E_{\mathrm{AFM}}(0)}=\frac{t\left(\Delta G_{S}\right)}{t(0)} \approx \frac{1}{1+\Delta G_{S} / G_{\mathrm{AFM}}} .
$$

The last approximation is justified because $Z_{0} G_{\mathrm{AFM}} \approx 15$ is significantly larger than $n_{A}+n_{S} \approx 4.6$ and approximately constant at 0-2 $\mathrm{THz}$ (see Appendix A). Photoinduced changes $\Delta G_{\mathrm{AFM}}$ in the conductance of the CuMnAs film are neglected because for below-gap excitation, we do not observe a sizeable modification of the terahertzinduced resistance modulation [Fig. 3(a)]. The photoinduced conductance $\Delta G_{S}$ equals the number of photogenerated electron-hole pairs in $\mathrm{GaAs}$ or $\mathrm{GaP}$ times their mobility $\mu$ and the elementary charge $e$. Thus, directly after arrival of the pump pulses, when charge-carrier recombination is not yet relevant, $\Delta G_{S}$ scales with the gate fluence $F_{\mathrm{opt}}$ and can be estimated using the relationship

$$
\Delta G_{S}=B F_{\mathrm{opt}} \text { with } B=\frac{e \mu T_{\mathrm{opt}}}{\epsilon_{\mathrm{opt}}} .
$$

The gate-intensity transmittance $T_{\mathrm{opt}}$ from air through the CuMnAs film to the substrate layer is calculated by the Airy formula that takes all reflection echoes in the CuMnAs into account, using the optical refractive indices of substrate, air, and CuMnAs [38] (see Appendix A). The relevant parameter values of the $\mathrm{GaAs}$ and $\mathrm{GaP}$ substrates are summarized in Table I.

We fit Eqs. (2) and (3) to the experimentally determined $C^{2}$ vs $F_{\text {opt }}$ by using proportionality constant $B$ as the only free parameter. As seen in Fig. 4(b) and 4(d), good agreement between model and experiment is obtained for $B=0.48 \mathrm{~S} \mathrm{~cm} / \mathrm{mJ}$ and $0.016 \mathrm{~S} \mathrm{~cm} / \mathrm{mJ}$ for the GaAs and $\mathrm{GaP}$ substrate, respectively. Using the extracted $B$ values, Eq. (3) and the relevant parameters at $\epsilon_{\text {opt }}=1.55 \mathrm{eV}$ (see Table I), we obtain a mobility of $\mu=5300 \mathrm{~cm}^{2} / \mathrm{V} \mathrm{s}$ of photoexcited electrons and holes in GaAs. These numbers are consistent with typical electron mobilities of photoexcited undoped GaAs substrates [31,41-45]. For GaP and $\epsilon_{\mathrm{opt}}=$ $3.1 \mathrm{eV}$, we obtain $\mu=1800 \mathrm{~cm}^{2} / \mathrm{V} \mathrm{s}$, whereas $400 \mathrm{~cm}^{2} / \mathrm{V} \mathrm{s}$ is an upper bound of reported photoinduced carrier mobilities in undoped GaP $[41,44,46-49]$. Note that the extracted value depends on the gate transmittance $T_{\text {opt }}$ through the CuMnAs layer and the square $b_{\mathrm{opt}}^{2}$ of the gate-beam radius. At $\epsilon_{\text {opt }}=3.1 \mathrm{eV}$, both $T_{\mathrm{opt}}$ and $b_{\mathrm{opt}}$ are estimated and can come with significant uncertainties of the order of $50 \%$. Therefore, we consider the obtained photocarrier mobility for $\mathrm{GaP}$ reasonably compatible with literature values.

This consistency supports our interpretation, suggesting that the gate-induced reduction of the resistance modulation of our samples arises from shunting by the photoconductance of optically generated charge carriers in the 
substrate [Fig. 1(c)]. Our model can in particular explain the much smaller gate power that is required to reduce the terahertz-resistance modulation of the sample with the GaAs [Fig. 4(b)] versus the GaP [Fig. 4(d)] substrate.

\section{DISCUSSION}

\section{A. Signal dynamics versus $\tau$}

Our interpretation of the gate-induced suppression of the resistance modulation [Fig. 1(c)] implies that the dynamics of $\Delta R$ as a function of the delay $\tau$ between the terahertz and optical gate pulse is given by the evolution of the transient substrate conductance $\Delta G_{S}$ [see Eq. (2)].

On longer scales $(\tau>1 \mathrm{ps})$, the temporal decay of $\Delta G_{S}$ and, thus, $\Delta R$ can arise from (i) diffusion of carriers out of the relevant substrate region and (ii) electron-hole recombination. Regarding (i), carrier diffusion is known to significantly change the optical response of nanophotonic devices [50] on a timescale of 100 ps. In our structure, carrier transport perpendicular to the film plane does not modify the transient substrate sheet conductance $\Delta G_{S}$. For in-plane diffusion, however, $\Delta G_{S}$ can undergo notable changes if the diffusion length becomes comparable to the smallest relevant structure size, which is the side length $(2-4 \mu \mathrm{m})$ of the square central region of our device [see Fig. 1(a)]. For GaAs, the diffusion constant $\left(D<200 \mathrm{~cm}^{2} / \mathrm{s}\right)[41,42,51,52]$ implies an upper limit to the diffusion length $\sqrt{D t}$ of $0.6,1.4$, and $2.8 \mu \mathrm{m}$ at times $t=20 \mathrm{ps}, 100$, and $400 \mathrm{ps}$, respectively. For GaP $(D<$ $10 \mathrm{~cm}^{2} / \mathrm{s}$ ) [41,46,53], the respective lengths amount to $0.14,0.32$, and $0.63 \mu \mathrm{m}$. Thus, for GaAs and on a timescale of $100 \mathrm{ps}$, carrier diffusion may well lead to a significant decay of $\Delta G_{S}$. However, the measured switching contrast $\Delta R$ exhibits a much slower decay [see Fig. 2(d)]. Possible reasons for this behavior are as follows. First, the relationship between $\Delta R$ and $P_{\text {opt }}$ and, thus, $\Delta G_{S}$ is highly nonlinear and becomes relatively insensitive to $P_{\text {opt }}$ and, thus, $\Delta G_{S}$ at the higher $P_{\text {opt }}$ used here [see Figs. 4(a) and 4(c)]. Second, the relevant length scale of our structure is larger than the assumed approximately $1 \mu \mathrm{m}$. Third, the diffusion constant of the GaAs substrate is much smaller than assumed. In contrast to GaAs, our previous estimates for $\mathrm{GaP}$ suggest that carrier diffusion makes a minor contribution to the decay of $\Delta G_{S}$ and, thus, $\Delta R$ [Fig. 3(b)].

Regarding (ii), we note that the relaxation times of $\Delta R$ seen in our experiments are compatible with timescales found in previous works. For GaAs-based samples, we find a monoexponential decay with time constant of $2.3 \mathrm{~ns}$, which agrees well with the typical range of carrier lifetimes in GaAs from several hundreds of picoseconds to nanoseconds, depending on the density of in-gap states and compensation doping [41,54-56]. For GaP, the time constants of the decay of $\Delta R$ vary from 140 to $800 \mathrm{ps}$, depending on excitation density. They are of the same order of magnitude as timescales found for photoexcited $\mathrm{GaP}[41,46,57-59]$ or other large-gap semiconductors such as $6 \mathrm{H}$-SiC [60] and $\mathrm{ZnTe}$ [61]. We emphasize that comparison of carrier lifetimes is useful only in a qualitative sense. First, they depend sensitively on sample properties such as defects, carrier density, and surface quality. Second, the observable $\Delta R$ considered here (Fig. 3) depends highly nonlinearly on the carrier density [see Figs. 4(a) and 4(c)] and so may not faithfully reflect the speed of the carrier decay.

For short timescales of around $\tau=0$, the switch-on dynamics of the transient photoconductance $\Delta G_{S}$ is given by the gate-pulse duration and the time it takes the photogenerated carriers to establish a Drude-type conductivity. As shown by previous works $[62,63]$, the timescale of the latter process is typically $50-100 \mathrm{fs}$, depending on excitation conditions.

At positive and negative delays $\tau$, the transverse resistance signal $R_{x y}$ exhibits a different offset [see Figs. 2(b) and 2(c)]. A possible origin of the offset is that spatial symmetries (such as mirror symmetries) of the ideal sample structure are slightly broken in the fabricated sample, for instance due to nonorthogonal contacts, anisotropic conductivity of CuMnAs [23], and an asymmetric terahertzbeam cross section. As a consequence, the sample is neither switched nor read out in a spatially symmetric manner, even in the absence of the optical gate pulse. Therefore, the sum of resistances of the states obtained by $\pm 45^{\circ}$-oriented electric fields can change. Another reason for the offset change of $R_{x y}$ may be the possible existence of slowly relaxing signal components, which can be found to last for tens of minutes after switching [6]. The offset change mostly cancels in the switching contrast $\Delta R$ [Fig. 2(b)].

\section{B. Other gate-pulse excitations}

In our experiment, a substantial part of the gate pulse is absorbed by the AFM CuMnAs film. As shown by Figs. 2(d) and 3 for $\tau<0$, the gate pulse is not found to modify the already written resistance state. We conclude that the gate-pulse energy densities used here do not reach the threshold that is needed for optical writing and erasing of resistance states in CuMnAs. Notable changes would require a transient increase of the $\mathrm{CuMnAs}$ temperature close to the Néel point [6]. In fact, we estimate the maximal energy density deposited by one optical pulse in the CuMnAs film in our experiment to be roughly $50 \mathrm{pJ} / \mu \mathrm{m}^{3}$, which is approximately 1 order of magnitude smaller than the densities used for optical writing and erasing in Ref. [6]. By comparison with these switching experiments, we estimate that the transient temperature increase of CuMnAs reaches a few $10 \mathrm{~K}$. 
The deposited optical gate-pulse energy could still ease the terahertz-pulse-driven switching by lowering the writing threshold and, thus, lead to an observable increase of the switching contrast $\Delta R$. However, the measurements [Fig. 3(a)] are in contrast to this expectation: They indicate a weak opposite trend, a slight decrease of the contrast. Therefore, we consider the optical gate-pulse energies used here to be optimal. We control the terahertz field strength in the CuMnAs film without directly changing the resistance state of the CuMnAs film.

The small signal difference between positive and negative delays at the highest gate fluence in Fig. 3(a) is assigned to two-photon absorption (2PA) in the GaP substrate. Indeed, estimates based on the $2 \mathrm{PA}$ coefficient of $\mathrm{GaP}[46,64,65]$ (see Appendix A) indicate that at the highest fluence $F_{\mathrm{opt}}^{\max }$ at $1.55 \mathrm{eV}$, the gate can reduce the terahertz field by a few per cent, i.e., $1-C \sim 10^{-2}$, and, thus, cause a small yet notable reduction of the switching contrast as suggested by Fig. 3(a).

We finally note that the gate-induced free charge carriers do not only modulate the electric component of the terahertz pulse. They may also lead to changes in the terahertz magnetic field, which are, however, not relevant for this experiment.

\section{Possible functionality and power consumption}

In a wider application perspective, our results demonstrate the integration of an AFM memory with a powerful semiconductor functionality. They suggest a pathway to achieve terahertz-field-induced resistance switching of selected regions of the CuMnAs film, an application which is analogous to an "optical-gate transistor". Illumination with a structured gate-beam cross section protects the illuminated regions from being switched by the global terahertz field without affecting the already stored information. For free-space gate beams, the smallest feature size and, thus, footprint of a bit is given by approximately half the gate wavelength, that is, $\lambda_{\text {opt }} / 2=200 \mathrm{~nm}$. This value is 3 orders of magnitude smaller than the terahertz beam diameter. Adequate and rapid structuring of light beams can be achieved with binary spatial light modulators [66]. Even smaller feature sizes of the order of $50 \times 50 \mathrm{~nm}^{2}$ are routinely possible with optical nanoantennas [67].

The integration of AFM memory functionality with logical features of semiconductors shown here and the compatibility of CuMnAs with various semiconductor substrates open the door toward a rich variety of applications. For instance, an optical excitation of memory bits, similar to our optical protection from terahertz writing, is already successfully employed in heat-assisted magnetic recording (HAMR) technology [67]. Here, a magnetically hard ferromagnetic recording surface is exposed to a magnetic field of relatively large spatial extent, but the data is written only in a small region softened by thermal heating from an optical beam.

It is interesting to compare the optical energy required to soften one magnetic bit in HAMR to the energy that would be required to excite the semiconducting substrate and protect one bit from terahertz-field-induced writing. Assuming a bit size [67] of $50 \times 50 \mathrm{~nm}^{2}$, we estimate that HAMR requires an optical energy of $30 \mathrm{pJ} / \mathrm{bit}[68,69]$. This value is more than 5 orders of magnitude larger than the $0.5 \mathrm{fJ} /$ bit needed for bit protection in our samples (see Appendix B). This comparison should be taken with caution. First, our scheme is an inverse of HAMR because we need to protect a bit, which should remain unchanged, every time it is inside an area that is affected by the extended terahertz field. Second, at room temperature, bits in our CuMnAs device are 5-6 orders of magnitude shorter lived than in ferromagnetic memories. Nevertheless, our energy comparison shows that the scheme suggested in this work [Fig. 1(c)] is comparatively energy efficient and takes the advantages of AFM data storage to semiconductor electronics.

The timescale over which the AFM memory stays protected against the terahertz switching is determined by the lifetime of the photoinduced carriers. The latter can be set over a wide range from subpicoseconds to nanoseconds by standard semiconductor methods, for example control of the impurity concentration and growth temperature of the substrate $[70,71]$.

\section{CONCLUSION}

Our results demonstrate an efficient control of the terahertz switching of a CuMnAs AFM layer by functionalizing the semiconducting substrate. When excited with an optical pulse, the substrate becomes conducting and shorts the terahertz writing current in the AFM layer. A parallel-photoconductor model can quantitatively explain our findings. Notably, we do not observe a direct influence of the optical gate absorption by the AFM layer itself. For technological applications, the lifetime of the photoinduced changes in the conductivity of the semiconducting substrate can be tuned by doping and defect density. The shown integration of AFM memory functionality with logical features of semiconductors and the compatibility of $\mathrm{CuMnAs}$ with various semiconductor substrates open the door toward a rich variety of applications known from dilute magnetic semiconductors [72,73] and multiferroic spintronics [74].

\section{ACKNOWLEDGMENTS}

We thank the European Union for support through the projects CoG TERAMAG/Grant No. 681917 and ASPIN/Grant No. 766566, the Ministry of Education of the Czech Republic for Grant No. LM2018110 and LNSM-LNSpin, the Czech Science Foundation (GA CR) 
for Grant No. 21-28876J, and the German Research Foundation for funding through the collaborative research center SFB TRR 227 "Ultrafast spin dynamics" (Project No. A05). J.J.F. Heitz acknowledges support by the IMPRS for Elementary Processes in Physical Chemistry.

\section{APPENDIX A: MODEL OF PARALLEL CONDUCTIVITY}

\section{Optical transmittance}

The gate-intensity transmittance $T_{\mathrm{opt}}$ from air through the CuMnAs film to the substrate layer, as it appears in Eq. (3), is given by the relationship $T_{\mathrm{opt}}=\left(|t|^{2} \operatorname{Re} n_{\mathrm{S}} /\right.$ $\left.\operatorname{Re} n_{\mathrm{A}}\right)_{\mathrm{opt}}$, where the index opt indicates that all quantities need to be evaluated for the optical gate. The transmission coefficient $t_{\mathrm{opt}}$ of the gate field from air through the CuMnAs film into the substrate is given by [75]

$$
t_{\mathrm{opt}}=\frac{t_{12} t_{23} \exp \left(2 \pi i n_{2} d / \lambda\right)}{1+r_{12} r_{23} \exp \left(4 \pi i n_{2} d / \lambda\right)},
$$

where $t_{i j}$ and $r_{i j}$ are the Fresnel transmission and reflection coefficients of the interface of layers $i$ and $j$ for normal incidence (1: air, 2: CuMnAs, 3: substrate), $\lambda$ is the gate wavelength and $d=50 \mathrm{~nm}$ is the thickness of CuMnAs, all evaluated at the relevant gate frequency using the optical refractive indices of substrate $\left(n_{S, \text { opt }}\right)$, air $\left(n_{A, \text { opt }}\right)$, and CuMnAs $\left(n_{\mathrm{AFM}, \mathrm{opt}}=3.7+2.0 i\right.$ and $2.5+2.2 i$ at $\lambda=$ $800 \mathrm{~nm}$ and $400 \mathrm{~nm}$, respectively) [38].

The gate-intensity absorptance $A_{\mathrm{opt}}=1-T_{\mathrm{opt}}-R_{\mathrm{opt}}$ in the CuMnAs layer on top of the GaP substrate is obtained through an analogous expression for the reflectance, $R_{\mathrm{opt}}=$ $\left|r_{\text {opt }}\right|^{2}$ with [75]

$$
r_{\mathrm{opt}}=\frac{r_{12}+r_{23} \exp \left(4 \pi i n_{2} d / \lambda\right)}{1+r_{12} r_{23} \exp \left(4 \pi i n_{2} d / \lambda\right)} .
$$

\section{Terahertz conductivity of CuMnAs}

The conductance of the 50-nm thin CuMnAs films on GaAs and GaP substrates used in Eq. (2) is obtained by dc electrical measurements of the actual devices used in the terahertz experiment and yields $G_{\mathrm{AFM}} \approx 40$ and $50 \mathrm{mS}$, respectively. The reliability of this value at terahertz frequencies is confirmed by a terahertz conductivity measurement on a $20-\mathrm{nm}$ thin unpatterned CuMnAs film on a GaP substrate (see Fig. S1 in the Supplemental Material [33]). The conductivity is approximately constant in the relevant frequency range from 0.1 to $2 \mathrm{THz}$ and amounts to $\sigma \approx 8 \times 10^{5} \mathrm{~S} / \mathrm{m}$, yielding a conductance of approximately $40 \mathrm{mS}$ for a $50-\mathrm{nm}$ thin film, which is roughly consistent with the dc values used.

\section{Terahertz penetration depths}

The penetration depth $\delta$ of terahertz radiation at $1 \mathrm{THz}$ is roughly estimated using the relation $[76,77]$

$$
\delta(\omega) \approx \sqrt{\frac{2 c}{Z_{0} \sigma(\omega) \omega}},
$$

where $\omega / 2 \pi=1 \mathrm{THz}, Z_{0}=377 \Omega$, and $c$ is the light velocity in a vacuum. It yields $\delta \approx 560 \mathrm{~nm}$ for CuMnAs using $\sigma(\omega) \approx 8 \times 10^{5} \mathrm{~S} / \mathrm{m}$.

To estimate $\delta$ of the optically excited substrates, we assume the photoconductance $\Delta G_{S}$ is much higher than the residual conductivity of semi-insulating GaAs and $\mathrm{GaP}$. Then, using Eq. (2), we express the photoconductivity of the substrate in terms of $C$ by $\Delta \sigma_{S}=G_{\mathrm{AFM}}\left(C^{-1}-1\right) / \delta_{S}^{\mathrm{opt}}$ where $G_{\mathrm{AFM}}=40$ and $50 \mathrm{mS}$ and $\delta_{S}^{\mathrm{opt}}=750$ and $116 \mathrm{~nm}$ are the conductances and the optical penetration depths of $\mathrm{GaAs}$ and $\mathrm{GaP}$ at wavelengths of 800 and $400 \mathrm{~nm}$, respectively. We use $C$ values for the highest optical excitation from Fig. 4 and replace $\sigma$ by $\Delta \sigma_{S}$ in Eq. (A3), obtaining the terahertz skin depth $\delta \approx 4.9$ and $1.0 \mu \mathrm{m}$ for GaAs and $\mathrm{GaP}$, respectively. As $\delta_{\mathrm{AFM}}^{\mathrm{THz}}$ and $\delta_{S}^{\mathrm{THz}}$ are much larger than the thickness of the CuMnAs film and the depth of the photoexcitation in the substrates, the use of the Tinkham formula in Eq. (1) of the main text is appropriate.

\section{Two-photon absorption in GaP}

The optical intensity $I_{\mathrm{abs}}$ absorbed in a sheet at depth $z$ in the transparent GaP substrate uniquely by $2 \mathrm{PA}$ is $[64,78]$

$$
I_{\mathrm{abs}}(z)=I_{0}-I(z)=I_{0}\left(1-\frac{1}{1+z \beta I_{0}}\right)
$$

where $I_{0}$ is the intensity at $z=0$ and $\beta$ is the $2 \mathrm{PA}$ coefficient defined by the differential equation $\partial I / \partial z=-\beta I^{2}$. Using the typical value $\beta=1 \mathrm{~cm} / \mathrm{GW}[38,46,65]$ and the peak intensity $I_{0}=T_{\mathrm{opt}} F_{\mathrm{opt}}^{\max } / \tau_{p}$ of our maximal excitation at the below-band-gap excitation energy $1.55 \mathrm{eV}$ and pulse duration $\tau_{p}=40 \mathrm{fs}$, we obtain the absorbed fraction of the gate intensity $I_{\mathrm{abs}}(z) / I_{0} \approx 0.2 \%, 0.9 \%$, and $1.8 \%$ for the excitation depths of $z=1,5$ and $10 \mu \mathrm{m}$, respectively. These values already indicate that $2 \mathrm{PA}$ can induce an observable transient photoconductance $\Delta G_{S}$.

Using the absorbed intensity and Eq. (3) of the main text, we can express the equivalent gate fluence $F_{\mathrm{opt}}^{\mathrm{eq}}$ at the above-band-gap energy $3.1 \mathrm{eV}$ which corresponds to the same $\Delta G_{S}$. We obtain $F_{\text {opt }}^{\text {eq }} / F_{\text {opt }}^{\max }(3.1 \mathrm{eV})=0.5 \%, 2.3 \%$ and $4.6 \%$, respectively. By comparison to Fig. 3(a) and 3 (b) of the main text, we conclude that $2 \mathrm{PA}$ in $\mathrm{GaP}$ could explain the observed slight reduction of the switching signal at the below-band-gap gate excitation. 


\section{APPENDIX B: WRITING-ENERGY REQUIREMENT IN HAMR}

To compare the energy requirement of writing a bit of data in HAMR media to the energy requirement of protecting a bit in the scheme presented here, one needs to consider the following. In both HAMR and the technique presented in this work, the excitation is pulselike, and a single bit is excited for less than $10 \mathrm{~ns}$ in each case.

The HAMR scheme described in Ref. [69] employs a plasmonic near-field transducer (NFT) to focus energy from a diode (wavelength $830 \mathrm{~nm}$, peak power $8.5 \mathrm{~mJ} / \mathrm{s}$ ) into a $50 \mathrm{~nm} \times 50 \mathrm{~nm}$ region, beating the diffraction limit. The NFT can be optimized to deliver approximately $57 \%$ of the incident energy to the ferromagnetic recording layer. Thus, effectively $4.8 \mathrm{~mJ} / \mathrm{s}$ are available. Assuming that the drive writes a continuous stream of $50 \times 50 \mathrm{~nm}^{2}$ bits at a linear speed of $7.2 \mathrm{~m} / \mathrm{s}$ as the disk in Ref. [68], a rate of $7.2 / 50 \times 10^{-9} \mathrm{bit} / \mathrm{s}=144 \mathrm{Mbit} / \mathrm{s}$ results, corresponding to an exposition on each bit for about $7 \mathrm{~ns}$. For continuous excitation, this value implies that $4.8 \mathrm{~mJ} / 144 \mathrm{Mbit}=$ $33 \mathrm{pJ} / \mathrm{bit}$ are required for writing to the disk.

In the scheme presented here, however, we excite a circular $85-\mu \mathrm{m}$-diameter area (Table I) of the semiconducting substrate with approximately $2 \mu \mathrm{W}$ of $800 \mathrm{~nm}$ light [Fig. 4(a)]. At $1 \mathrm{kHz}$ repetition rate, this condition corresponds to $2 \mathrm{~nJ}$ energy distributed over this $5670 \mu \mathrm{m}^{2}$ area, which, considering the energy deposited in a $50 \times 50 \mathrm{~nm}^{2}$ area, is equivalent to $0.9 \mathrm{fJ} / \mathrm{bit}$. By functionalizing the semiconducting substrate instead of heating the data layer itself, 5 orders of magnitude less energy per bit are needed.

[1] M. B. Jungfleisch, W. Zhang, and A. Hoffmann, Perspectives of antiferromagnetic spintronics, Phys. Lett. A 382, 865 (2018).

[2] S. F. Maehrlein, I. Radu, P. Maldonado, A. Paarmann, M. Gensch, A. M. Kalashnikova, R. V. Pisarev, M. Wolf, P. M. Oppeneer, J. Barker, and T. Kampfrath, Dissecting spinphonon equilibration in ferrimagnetic insulators by ultrafast lattice excitation, Sci. Adv. 4, eaar5164 (2018).

[3] C. Kittel, Theory of antiferromagnetic resonance, Phys. Rev. 82, 565 (1951).

[4] T. Kampfrath, A. Sell, G. Klatt, A. Pashkin, S. Mährlein, T. Dekorsy, M. Wolf, M. Fiebig, A. Leitenstorfer, and R. Huber, Coherent terahertz control of antiferromagnetic spin waves, Nat. Photon 5, 31 (2011).

[5] C. Song, Y. You, X. Chen, X. Zhou, Y. Wang, and F. Pan, How to manipulate magnetic states of antiferromagnets, Nanotechnology 29, 112001 (2018).

[6] Z. Kašpar, M. Surýnek, J. Zubáč, F. Krizek, V. Novák, R. P. Campion, M. S. Wörnle, P. Gambardella, X. Marti, P. Němec, K. W. Edmonds, S. Reimers, O. J. Amin, F. Maccherozzi, S. S. Dhesi, P. Wadley, J. Wunderlich, K. Olejník, and T. Jungwirth, Quenching of an antiferromagnet into high resistivity states using electrical or ultrashort optical pulses, Nat. Electron. 4, 30 (2020).
[7] M. Wang, et al., Spin flop and crystalline anisotropic magnetoresistance in CuMnAs, Phys. Rev. B 101, 094429 (2020).

[8] S. Wienholdt, D. Hinzke, and U. Nowak, THz Switching of Antiferromagnets and Ferrimagnets, Phys. Rev. Lett. 108, 247207 (2012).

[9] P. Wadley, B. Howells, J. Železný, C. Andrews, V. Hills, R. P. Campion, V. Novák, K. Olejník, F. Maccherozzi, and S. S. Dhesi et al., Electrical switching of an antiferromagnet, Science 351, 587 (2016).

[10] P. Wadley, S. Reimers, M. J. Grzybowski, C. Andrews, M. Wang, J. S. Chauhan, B. L. Gallagher, R. P. Campion, K. W. Edmonds, S. S. Dhesi, F. Maccherozzi, V. Novak, J. Wunderlich, and T. Jungwirth, Current polarity-dependent manipulation of antiferromagnetic domains, Nat. Nanotech. 13, 362 (2018).

[11] J. Godinho, H. Reichlová, D. Kriegner, V. Novák, K. Olejník, Z. Kašpar, Z. Šobáň, P. Wadley, R. P. Campion, R. M. Otxoa, P. E. Roy, J. Železný, T. Jungwirth, and J. Wunderlich, Electrically induced and detected néel vector reversal in a collinear antiferromagnet, Nat. Commun. 9, 4686 (2018).

[12] P. E. Roy, R. M. Otxoa, and J. Wunderlich, Robust picosecond writing of a layered antiferromagnet by staggered spin-orbit fields, Phys. Rev. B 94, 014439 (2016).

[13] S. Schlauderer, C. Lange, S. Baierl, T. Ebnet, C. P. Schmid, D. C. Valovcin, A. K. Zvezdin, A. V. Kimel, R. V. Mikhaylovskiy, and R. Huber, Temporal and spectral fingerprints of ultrafast all-coherent spin switching, Nature 569, 383 (2019).

[14] T. Dannegger, M. Berritta, K. Carva, S. Selzer, U. Ritzmann, P. M. Oppeneer, and U. Nowak, Ultrafast coherent all-optical switching of an antiferromagnet with the inverse Faraday effect, Phys. Rev. B 104, L060413 (2021).

[15] S. Yu. Bodnar, L. Šmejkal, I. Turek, T. Jungwirth, O. Gomonay, J. Sinova, A. A. Sapozhnik, H.-J. Elmers, M. Kläui, and M. Jourdan, Writing and reading antiferromagnetic $\mathrm{Mn}_{2} \mathrm{Au}$ by néel spin-orbit torques and large anisotropic magnetoresistance, Nat. Commun. 9, 348 (2018).

[16] S. Yu. Bodnar, Y. Skourski, O. Gomonay, J. Sinova, M. Kläui, and M. Jourdan, Magnetoresistance Effects in the Metallic Antiferromagnet $\mathrm{Mn}_{2} \mathrm{Au}$, Phys. Rev. Appl. 14, 014004 (2020).

[17] J. Železný, H. Gao, K. Výborný, J. Zemen, J. Mašek, A. Manchon, J. Wunderlich, J. Sinova, and T. Jungwirth, Relativistic Néel-Order Fields Induced by Electrical Current in Antiferromagnets, Phys. Rev. Lett. 113, 157201 (2014).

[18] K. Olejník, V. Schuler, X. Marti, V. Novák, Z. Kašpar, P. Wadley, R. P. Campion, K. W. Edmonds, B. L. Gallagher, J. Garces, M. Baumgartner, P. Gambardella, and T. Jungwirth, Antiferromagnetic CuMnAs multi-level memory cell with microelectronic compatibility, Nat. Commun. 8, 15434 (2017).

[19] M. S. Wörnle, P. Welter, Z. Kašpar, K. Olejník, V. Novák, R. P. Campion, P. Wadley, T. Jungwirth, C. L. Degen, and P. Gambardella, Current-induced fragmentation of antiferromagnetic domains, ArXiv:1912.05287 (2019).

[20] S. Manz, M. Matsubara, T. Lottermoser, J. Büchi, A. Iyama, T. Kimura, D. Meier, and M. Fiebig, Reversible 
optical switching of antiferromagnetism in $\mathrm{TbMnO}_{3}$, Nature Photon 10, 653 (2016).

[21] T. Matalla-Wagner, M.-F. Rath, D. Graulich, J.-M. Schmalhorst, G. Reiss, and M. Meinert, Electrical Néel-Order Switching in Magnetron-Sputtered CuMnAs Thin Films, Phys. Rev. Appl. 12, 064003 (2019).

[22] K. Olejník, T. Seifert, Z. Kašpar, V. Novák, P. Wadley, R. P. Campion, M. Baumgartner, P. Gambardella, P. Němec, J. Wunderlich, J. Sinova, P. Kužel, M. Müller, T. Kampfrath, and T. Jungwirth, Sci. Adv. 4, eaar3566 (2018).

[23] F. Krizek, Z. Kašpar, A. Vetushka, D. Kriegner, E. M. Fiordaliso, J. Michalicka, O. Man, J. Zubáč, M. Brajer, V. A. Hills, K. W. Edmonds, P. Wadley, R. P. Campion, K. Olejník, T. Jungwirth, and V. Novák, Molecular beam epitaxy of CuMnAs, Phys. Rev. Mater. 4, 014409 (2020).

[24] R. Ulbricht, E. Hendry, J. Shan, T. F. Heinz, and M. Bonn, Carrier dynamics in semiconductors studied with timeresolved terahertz spectroscopy, Rev. Mod. Phys. 83, 543 (2011).

[25] J. Lloyd-Hughes and T. I. Jeon, A review of the terahertz conductivity of bulk and nano-materials, J. Infrared, Millimeter, Terahertz Waves 33, 871.

[26] Y. Shi, Q. Zhoua, C. Zhang, and B. Jin, Ultrafast high-field carrier transport in GaAs measured by femtosecond pumpterahertz probe spectroscopy, Appl. Phys. Lett. 93, 121115 (2008).

[27] H. T. Chen, J. F. O’Hara, A. K. Azad, and A. J. Taylor, Manipulation of terahertz radiation using metamaterials, Laser Photonics Rev. 5, 513 (2011).

[28] T. Okada, K. Ooi, Y. Nakata, K. Fujita, K. Tanaka, and K. Tanaka, Direct creation of a photoinduced metallic structure and its optical properties in the terahertz frequency region, Opt. Lett. 35, 1719 (2010).

[29] N. Kamaraju, A. Rubano, L. Jian, S. Saha, T. Venkatesan, J. Nötzold, R. K. Campen, M. Wolf, and T. Kampfrath, Subcycle control of terahertz waveform polarization using alloptically induced transient metamaterials, Nature: Light: Sci. Appl. 3, e155 (2014).

[30] M. B. Panish and H. C. Casey, Temperature dependence of the energy gap in GaAs and GaP, J. Appl. Phys. 40, 163 (1969).

[31] J. S. Blakemore, Semiconducting and other major properties of gallium arsenide, J. Appl. Phys. 53, R123 (1982).

[32] M. Sajadi, M. Wolf, and T. Kampfrath, Terahertz-fieldinduced optical birefringence in common window and substrate materials, Opt. Express 23, 28985 (2015).

[33] See Supplemental Material at http://ink.aps.org/supple mental/10.1103/PhysRevApplied.16.064047 for a detailed description of optical and electronic measurement setups and the characterization of optical and terahertz beams.

[34] J. Hebling, A. G. Stepanov, G. Almási, B. Bartal, and J. Kuhl, Tunable $\mathrm{THz}$ pulse generation by optical rectification of ultrashort laser pulses with tilted pulse fronts, Appl. Phys. B: Lasers Opt. 78, 593 (2004).

[35] D. Grischkowsky, S. Keiding, M. van Exter, and Ch. Fattinger, Far-infrared time-domain spectroscopy with terahertz beams of dielectrics and semiconductors, J. Opt. Soc. Am. B 7, 2006 (1990).
[36] K. L. Vodopyanov and Yu. H. Avetisyan, Optical terahertz wave generation in a planar GaAs waveguide, Opt. Lett. 33, 2314 (2008).

[37] D. E. Aspnes and A. A. Studna, Dielectric functions and optical parameters of $\mathrm{Si}, \mathrm{Ge}, \mathrm{GaP}, \mathrm{GaAs}, \mathrm{GaSb}, \mathrm{InP}$, InAs, and InSb from 1.5 to $6.0 \mathrm{eV}$, Phys. Rev. B 27, 985 (1983).

[38] M. Veis, J. Minár, G. Steciuk, L. Palatinus, C. Rinaldi, M. Cantoni, D. Kriegner, K. K. Tikuišis, J. Hamrle, and M. Zahradník et al., Band structure of CuMnAs probed by optical and photoemission spectroscopy, Phys. Rev. B 97, 125109 (2018).

[39] J. Neu, K. P. Regan, J. R. Swierk, and C. A. Schmuttenmaer, Applicability of the thin-film approximation in terahertz photoconductivity measurements, Appl. Phys. Lett. 113, 233901 (2018).

[40] L. Nádvorník, M. Borchert, L. Brandt, R. Schlitz, K. A. de Mare, K. Výborný, I. Mertig, G. Jakob, M. Kläui, S. T. B. Goennenwein, M. Wolf, G. Woltersdorf, and T. Kampfrath, Broadband Terahertz Probes of Anisotropic Magnetoresistance Disentangle Extrinsic and Intrinsic Contributions, Phys. Rev. X 11, 021030 (2021).

[41] B. G. Alberding, W. Robert Thurber, and E. J. Heilweil, Direct comparison of time-resolved terahertz spectroscopy and Hall Van Der Pauw methods for measurement of carrier conductivity and mobility in bulk semiconductors, J. Opt. Soc. Am. B 34, 1392 (2017).

[42] T. J. Magnanelli and E. J. Heilweil, Carrier mobility of silicon by sub-bandgap time-resolved terahertz spectroscopy, Opt. Express 28, 7221 (2020).

[43] J. N. Heyman, D. Bell, and T. Khumalo, Terahertz photoHall measurements of carrier mobility in GaAs and InP, Appl. Phys. Lett. 88, 162104 (2006).

[44] D. L. Rode, in Semiconductors and Semimetals, edited by R. K. Willardson and A. C. Beer (Elsevier, Amsterdam, Boston, 1975), Vol. 10, pp.1-89.

[45] G. E. Stillman, C. M. Wolfe, and J. O. Dimmock, Hall coefficient factor for polar mode scattering in N-type GaAs, J. Phys. Chem. Solids 31, 1199 (1970).

[46] J. K. Wahlstrand and E. J. Heilweil, Contactless THzbased bulk semiconductor mobility measurements using two-photon excitation, Opt. Express 26, 29848 (2018).

[47] H. C. Casey, F. Ermanis, and K. B. Wolfstirn, Variation of electrical properties with $\mathrm{Zn}$ concentration in GaP, J. Appl. Phys. 40, 2945 (1969).

[48] Y. C. Kao and O. Eknoyan, Electron and hole carrier mobilities for liquid phase epitaxially grown $\mathrm{GaP}$ in the temperature range 200-550 K, J. Appl. Phys. 54, 2468 (1983).

[49] J. D. Wiley, in Semiconductors and Semimetals, edited by R. K. Willardson and A. C. Beer (Elsevier, Amsterdam, Boston, 1975), Vol. 10, pp.91-174.

[50] D. M. Beggs, T. F. Krauss, L. Kuipers, and T. Kampfrath, Phys. Rev. Lett. 108, 033902 (2012).

[51] J. Požela and A. Reklaitis, Diffusion coefficient of hot electrons in GaAs, Solid State Commun. 27, 1073 (1978).

[52] J. G. Ruch and G. S. Kino, Transport properties of GaAs, Phys. Rev. 174, 921 (1968).

[53] M. L. Young and D. R. Wight, Concentration dependence of the minority carrier diffusion length and lifetime in GaP, J. Phys. D: Appl. Phys. 7, 1824 (1974). 
[54] M. Niemeyer, P. Kleinschmidt, A. W. Walker, L. E. Mundt, C. Timm, R. Lang, T. Hannappel, and D. Lackner, Measurement of the non-radiative minority recombination lifetime and the effective radiative recombination coefficient in GaAs, AIP Adv. 9, 045034 (2019).

[55] F. Cadiz, D. Lagarde, P. Renucci, D. Paget, T. Amand, H. Carrère, A. C. H. Rowe, and S. Arscott, Spin and recombination dynamics of excitons and free electrons in p-type GaAs: Effect of carrier density, Appl. Phys. Lett. 110, 082101 (2017).

[56] L. H. Teng, K. Chen, J. H. Wen, W. Z. Lin, and T. S. Lai, Density dependence of electron-spin polarization and relaxation in intrinsic GaAs at room temperature, J. Phys. D: Appl. Phys. 42, 135111 (2009).

[57] G. Lin, Q. Zhang, X. Lin, D. Zhao, R. Jia, N. Gao, Z. Zuo, $\mathrm{X}$. $\mathrm{Xu}$, and D. Liu, Enhanced photoluminescence of gallium phosphide by surface plasmon resonances of metallic nanoparticles, RSC Adv. 5, 48275 (2015).

[58] C. M. Collier, B. Born, X. Jin, and J. F. Holzman, Ultrafast charge-carrier and phonon dynamics in GaP, Appl. Phys. Lett. 103, 072106 (2013).

[59] M. A. Cavicchia and R. R. Alfano, Time-resolved IRabsorption spectroscopy of hot-electron dynamics in satellite and upper conduction bands in GaP, Phys. Rev. B 51, 9629 (1995).

[60] A. Rubano, M. Wolf, and T. Kampfrath, Terahertz conductivity and ultrafast dynamics of photoinduced charge carriers in intrinsic 3C and 6H silicon carbide, Appl. Phys. Lett. 105, 032104 (2014).

[61] M. Sajadi, M. Wolf, and T. Kampfrath, Terahertz field enhancement via coherent superposition of the pulse sequences after a single optical-rectification crystal, Appl. Phys. Lett. 104, 091118 (2014).

[62] R. Huber, F. Tauser, A. Brodschelm, M. Bichler, G. Abstreiter, and A. Leitenstorfer, How many-particle interactions develop after ultrafast excitation of an electron-hole plasma, Nature 414, 286 (2001).

[63] B. Mayer, C. Schmidt, J. Bühler, D. V. Seletskiy, D. Brida, A. Pashkin, and A. Leitenstorfer, Sub-cycle slicing of phase-locked and intense mid-infrared transients, New J. Phys. 16, 063033 (2014).

[64] V. Nathan, S. S. Mitra, and A. H. Guenther, Review of multiphoton absorption in crystalline solids, J. Opt. Soc. Am. B 2, 294 (1985).

[65] G. Grinblat, M. P. Nielsen, P. Dichtl, Y. Li, R. F. Oulton, and S. A. Maier, Ultrafast sub-30-Fs all-optical switching based on gallium phosphide, Sci. Adv. 5, eaaw3262 (2019).
[66] R. I. Stantchev, B. Sun, S. M. Hornett, P. A. Hobson, G. M. Gibson, M. J. Padgett, and E. Hendry, Noninvasive, near-field terahertz imaging of hidden objects using a single-pixel detector, Sci. Adv. 2, e1600190 (2016).

[67] D. Weller, G. Parker, O. Mosendz, A. Lyberatos, D. Mitin, N. Y. Safonova, and M. Albrecht, Review article: FePt heat assisted magnetic recording media, J. Vac. Sci. Technol. B 34, 060801 (2016).

[68] W. A. Challener, C. Peng, A. V. Itagi, D. KArns, W. Peng, Y. Peng, X. Yang, X. Zhu, N. J. Gokemeijer, Y.-T. Hsia, G. Ju, R. E. Rottmayer, M. A. Seigler, and E. C. Gage, Heat-assisted magnetic recording by a near-field transducer with efficient optical energy transfer, Nat. Photonics 3, 220 (2009).

[69] C. Zhong, P. Flanigan, N. Abadía, F. Bello, B. D. Jennings, G. Atcheson, J. Li, J.-Y. Zheng, J. J. Wang, R. Hobbs, D. McCloskey, and J. F. Donegan, Effective heat dissipation in an adiabatic near-field transducer for HAMR, Opt. Express 26, 18842 (2018).

[70] E. S. Harmon, M. R. Melloch, J. M. Woodall, D. D. Nolte, N. Otsuka, and C. L. Chang, Carrier lifetime versus anneal in low temperature growth GaAs, Appl. Phys. Lett. 63, 2248 (1993).

[71] V. A. Kozlov, F. Y. Soldatenkov, V. G. Danilchenko, V. I. Korolkov, and I. L. Shulpina, in 25th Annual SEMI Advanced Semiconductor Manufacturing Conference (ASMC 2014) (IEEE, Saratoga Springs, NY, 2014), pp. $139-144$.

[72] M. Tanaka, Recent progress in ferromagnetic semiconductors and spintronics devices, Jpn. J. Appl. Phys. 60, 010101 (2021).

[73] S. M. Yakout, Spintronics: Future technology for new data storage and communication devices, J Supercond Nov Magn 33, 2557 (2020).

[74] N. A. Spaldin and R. Ramesh, Advances in magnetoelectric multiferroics, Nature Mater 18, 203 (2019).

[75] P. Yeh, Optical Waves in Layered Media (Wiley, Hoboken, NJ, 2005).

[76] A. Abohmra, H. Abbas, M. Al-Hasan, I. B. Mabrouk, A. Alomainy, M. A. Imran, and Q. H. Abbasi, Terahertz antenna array based on a hybrid perovskite structure, IEEE Open J. Antennas Propag. 1, 464 (2020).

[77] A. K. Azad and W. Zhang, Resonant terahertz transmission in subwavelength metallic hole arrays of sub-skin-depth thickness, Opt. Lett. 30, 2945 (2005).

[78] M. Rumi and J. W. Perry, Two-photon absorption: An overview of measurements and principles, Adv. Opt. Photon. 2, 451 (2010). 